\title{
Clinical outcomes among febrile children aged 2 to 59 months with negative malaria rapid diagnostic test results in Mchinji District, Malawi
}

\author{
Dyson Mwandama ${ }^{1}$, Chawanangwa Mwale ${ }^{1}$, Andrew Bauleni ${ }^{1}$, Themba Phiri ${ }^{1}$, Joseph Chisaka ${ }^{2}$, \\ Humphreys Nsona ${ }^{3}$, Don P. Mathanga ${ }^{1}$
}

1. Malaria Alert Centre, College of Medicine, University of Malawi, Blantyre, Malawi

2 Queen Elizabeth Central Hospital, Blantyre, Malawi

3. IMCI Unit, Ministry of Health, Lilongwe, Malawi

Correspondence: Dr Don P. Mathanga (dmathang@mac.medcol.mw)

\begin{abstract}
Background

Malawi malaria treatment guidelines recommend a definitive diagnosis, using a malaria rapid diagnostic test (mRDT), for all patients with fever or history of fever. Improving the management and outcomes of febrile children with negative mRDT results should be a priority.

Methods

Through a prospective cohort study designed to investigate clinical outcomes of children treated at the community level, we followed, for 7 days, children aged 2 to 59 months, who had negative mRDT results and were treated with antipyretic medication. Clinical outcomes were assessed on days 3 and 7 post-recruitment.

Results

The median age of recruited children was 19 months. Of the 285 children enrolled, 139 (48.8\%) were females. Of the children for whom data were available for analysis, $95 / 236(40.3 \%)$ had fever (temperature $\left.\geq 37.5^{\circ} \mathrm{C}\right)$ at enrolment, and almost half of the sick children $(125 / 268 ; 46.6 \%)$ had symptoms of upper respiratory tract infection. Most sick children $(89.6 \% ; 95 \%$ confidence interval, CI $=84.2$ to 93.3$)$ recovered, while $10.4 \%(95 \%$ CI: 6.7 to 15.8 ) were still sick by day 7 of follow-up. There were no deaths reported during the 7 days of follow-up. Being afebrile at enrolment (odds ratio, $\mathrm{OR}=2.5 ; 95 \% \mathrm{CI}=1.1$ to $6.0 ; \mathrm{P}=0.027)$ and sleeping under an insecticide-treated net (ITN) $(\mathrm{OR}=2.7 ; 95 \% \mathrm{CI}=1.2$ to $6.2 ; \mathrm{P}=0.011)$ were associated with recovery by day 7. In multivariable analysis, sleeping under an ITN the previous night was the only factor associated with recovery by day 7. Microscopy did not detect any malaria parasites in the blood of recruited children, at recruitment or on day 7.
\end{abstract}

Conclusions

In this community-level study, the majority of febrile children with negative mRDT results recovered within 7 days of health worker consultation for a febrile illness, having only taken antipyretics.

\section{Introduction}

The World Health Organization (WHO) now recommends that all persons, in all epidemiological settings, who are suspected of having malaria, should have a parasitological confirmation of diagnosis by either a malaria rapid diagnostic test (mRDT) or microscopy. ${ }^{1}$ As a result, mRDTs are now being integrated into community case management programmes ${ }^{2-4}$ to mitigate against parasite resistance to antimalarial drugs and reduce waste associated with presumptive treatment. ${ }^{5}$ However, although mRDTs are highly sensitive, ${ }^{6}$ health workers still treat for malaria even after negative mRDT test results. ${ }^{78}$ The fear that a child who has a false negative mRDT result may be sent home to deteriorate has been suggested as the reason why negative mRDT results are so often disregarded. This noncompliance to negative results is a threat to the success of integrated community case management (iCCM), as it could lead to overtreatment, as well as missed diagnoses of other febrile illnesses. ${ }^{9-11}$ In this study, we assessed the clinical outcomes of febrile under-five children, with negative mRDT results, who were treated by community health workers with antipyretics at village clinics in Mchinji District, Malawi.

\section{Methods}

\section{Study setting}

The study was done in Mchinji District, in the Central Region of Malawi. The district has a total population of 511,792 , of which $17 \%$ are under-five children. ${ }^{12}$ Childhood diseases are a serious public health problem in the district, wherein between January and October 2014, 50,092 under- five children were diagnosed with malaria, 43,338 with diarrhoea, and 34,530 with acute respiratory infections (ARI). ${ }^{13,14}$ Malaria transmission is perennial in the district, peaking during the rainy season (November to April), and a third of the children will have fever at any given time. ${ }^{13}$ Treatment of common childhood diseases such as malaria, pneumonia, and diarrhoea occurs at 12 health centres across the district, or by community health workers (CHWs) who operate the 124 village clinics scattered across the district. ${ }^{13}$ These government-paid CHWs undergo a six-day community case management course, during which they are meant to acquire the capacity and skills to provide treatment for common childhood diseases (fever as a proxy of malaria, "fast breathing" [pneumonia], and diarrhoea) and conduct referrals for children with danger signs, as dictated by iCCM guidelines. In 2014, the district implemented a pilot project aimed at integrating mRDTs and rectal artesunate into the iCCM guidelines. Details of the pilot have been reported elsewhere. ${ }^{15}$

\section{Study design}

We conducted a prospective cohort study among children aged 2 to 59 months old, with fever or a history of fever within the 48 hours preceding enrolment, who had sought care from CHWs and were assessed and found to be mRDTnegative during classification. Caregivers of sick children were asked to return to the village clinics on days 3 and 7 for clinical assessment. The Malawi College of Medicine Research and Ethics Committee (COMREC) reviewed and approved the study. 


\section{Data collection}

\section{Enrolment}

Using a standardised study tool, enrolment was done in September 2014 at 14 randomly selected village clinics within the areas that implemented the aforementioned iCCM pilot project. ${ }^{15}$ Children with fever or a history of fever within the previous 48 hours, who were mRDT-negative using Paracheck-Pf devices (Orchid Biomedical Systems, Bambolim, India), who had not received any antimalarial drugs during the two weeks prior to enrolment, and who had no danger signs (convulsions, repeated vomiting, lethargy, severe anaemia, loss of consciousness) were eligible for participation. After their parents or guardians provided written informed consent, all enrolled children were assigned unique identification numbers, and a study team member administered a questionnaire to the guardians of each child. In addition, a thick blood smear sample was obtained from each participant for malaria parasite detection under microscopy. Detailed map directions to the home of each patient were recorded, in case a patient did not return for follow-up. Each sick child was prescribed an antipyretic and advised to come for review on days 3 and 7 post-enrolment. Follow-up visits

On Day 3, clinical assessments were done and study staff administered a questionnaire. Possible follow-up classifications were recovered (defined as a resolution of symptoms, for example absence of fever, and a child that was feeding well), still sick (defined as persistence or worsening of symptoms), dead, and lost to follow-up. All of the children were asked to come again on day 7 for a final clinical evaluation. On day 7, a study team member conducted a final clinical assessment, administered a questionnaire, collected a thick smear for malaria parasitaemia analysis, and determined a clinical outcome. Participants who did not return to the village clinic on either day 3 or day 7 were followed up at their homes by the study team. A participant who was not located after three consecutive attempts was assigned an outcome of lost to follow-up.

\section{Laboratory}

Thick blood smears were collected on days 0 and 7 from the study participants and were double-read. The slides were stained with 3\% Giemsa for 45 minutes. A blood slide was considered negative after examining 500 white blood cells with no asexual parasites.

\section{Sample size determination}

The baseline prevalence of fever among children 2 to 59 months of age was $52.8 \%$ in Mchinji District. ${ }^{13}$ The incidence of mRDT-negativity among the febrile children between 2 and 59 months of age was estimated at $20 \%$ in the district. Using a power of $90 \%$, with a probability of committing a type 1 error being 5\%, and assuming 20\% loss to follow-up rate, a sample size of 280 febrile children (or those who were febrile in the preceding 48 hours) between 2 and 59 months of age was determined.

\section{Statistical analysis}

As with febrile under-five children assessed for any other illnesses, and considering that children with a negative mRDT result are expected to recover without the use of antimalarials, the main outcome of interest was recovery within 7 days of follow-up.

We conducted a per protocol analysis. A multivariable model was chosen to reflect all of the predictors that were associated with clinical outcomes. All analyses were performed using Stata (version 12). For all analyses, a P-value less than 0.05 was considered significant.

\section{Results}

Of the 285 children that were enrolled, 139 (48.8\%) were females. The median age was 19 months, with an interquartile range (IQR) of 23. Of the children for whom data were available for analysis, 95/236 (40.3\%) had fever (temperature $\geq 37.5^{\circ} \mathrm{C}$ ) at enrolment, and almost half of the sick children $(125 / 268 ; 46.6 \%)$ had other symptoms that were suggestive of upper respiratory tract infection. All of the children were treated with antipyretics but none of the children received antibiotics (Table 1). During the night prior to enrolment, $210 / 259(81.1 \%)$ of the children were reported, by their guardians, to have slept under an insecticide-treated net (ITN), and microscopy revealed that none of the children had malaria parasitaemia on day 0 or day 7. Among the enrolled children, no deaths were reported during the 7-day follow-up period.

On day 3 of follow-up, out of 213 that reported for review, $178(83.6 \%)$ of the children had recovered, compared to $35(16.4 \%)$ who were still sick. Being afebrile at the time of enrolment (odds ratio, $\mathrm{OR}=2.5 ; 95 \% \mathrm{CI}=1.1$ to 6.0 ) and sleeping under an ITN the night before enrolment (OR = 2.7; $95 \% \mathrm{CI}=1.2$ to 6.2 ) were associated with recovery on day 3 .

By day 7, 163 (89.6\%) of 182 children (who returned for follow-up and for whom data were available for analysis) had recovered, and $19(10.4 \%)$ were still sick. Only sleeping under an ITN $(\mathrm{OR}=2.7 ; 95 \% \mathrm{CI}=1.1$ to 7.8$)$ was associated with recovery by day 7 . In multivariable analysis, sleeping under an ITN (OR $=4.3 ; 95 \% \mathrm{CI}=1.3$ to 13.7 ) was the only factor associated with recovery at day 7 . Of the 19 children who remained sick on day 7,15 were reported to be in the same condition as they were at enrolment, while 4 were reportedly in worse condition than at the baseline assessment.

\section{Discussion}

Our study has shown that the majority (89.6\%) of febrile under-five children with negative mRDT results, who were treated with antipyretics by CHWs and sent home, recovered by day 7 of follow-up. None of the children that remained sick had any malaria parasites on day 7. Our findings are consistent with the WHO and Malawi government recommendation to treat only confirmed malaria cases with antimalarial medication, and to assess for other causes of fever in febrile children with negative mRDT results then treat accordingly. ${ }^{16}$ The findings from this study also support the Malawi iCCM guidelines for CHWs, reaffirm that most non-malarial fevers resolve within a few days, and demonstrate that the trained CHWs can assess and manage fever in children at the community level. ${ }^{17}$

Interestingly, we found that all children who were either well or sick by day 7 of follow-up had blood smears that were negative for malaria on microscopy on day 0 and day 7 of follow-up. This reinforces the fact that not all febrile children have malaria and encourages adherence to guidance about negative mRDT results at all levels of the healthcare delivery system. ${ }^{16,18}$ An evaluation of Paracheck-Pf devices at four health centres in Blantyre District found that the sensitivity for these mRDT devices was relatively high (91\%) for detecting any level of parasitaemia, despite the tests being conducted by healthcare workers with varying qualifications. ${ }^{10}$ Another study (conducted in the Central 
Table 1: Demographic, socioeconomic, and clinical characteristics of study participants presenting to Mchinji village clinics at enrollment, day 3 , and day 7 of follow-up

\begin{tabular}{|c|c|c|c|c|c|c|c|}
\hline \multirow{2}{*}{ Variable } & \multirow{2}{*}{$\begin{array}{c}\begin{array}{c}\text { Day 0 } \\
(\mathbf{N}=\mathbf{2 8 5})\end{array} \\
\text { Sick } \\
\text { n (\%) }\end{array}$} & \multicolumn{3}{|c|}{$\begin{array}{c}\text { Day } 3 \\
(\mathrm{~N}=\mathbf{2 1 3})\end{array}$} & \multicolumn{3}{|c|}{$\begin{array}{c}\text { Day } 7 \\
(\mathrm{~N}=182)\end{array}$} \\
\hline & & $\begin{array}{c}\text { Well }=178 \\
\text { n (\%) }\end{array}$ & $\begin{array}{c}\text { Sick }=35 \\
\text { n (\%) }\end{array}$ & P-value & $\begin{array}{c}\text { Well = } 163 \\
\text { n (\%) }\end{array}$ & $\begin{array}{c}\text { Sick }=19 \\
\text { n (\%) }\end{array}$ & P-value \\
\hline Median age in months (IQR) & $19(23)$ & $22(24)$ & $19(26)$ & 0.60 & $17(25)$ & $22(17)$ & 0.12 \\
\hline Mean weight in $\mathrm{kg} \pm \mathrm{SD}$ & $10.3 \pm 2.7)$ & $10.5 \pm 2.8)$ & $10.5 \pm 2.7)$ & 0.88 & $10.6 \pm 2.7$ & $9.9 \pm 3.0$ & 0.26 \\
\hline \multicolumn{8}{|l|}{ Sex } \\
\hline Female & $139(48.8)$ & $91(51.1)$ & $18(51.4)$ & 0.39 & $89(54.6)$ & $9(47.4)$ & 0.18 \\
\hline $\begin{array}{l}\text { Afebrile at enrolment (temperature } \\
<37.5^{\circ} \mathrm{C} \text { ) }\end{array}$ & $141 / 236(59.8)$ & $89 / 140(63.6)$ & $11 / 27(40.7)$ & 0.027 & $78 / 118(66.1)$ & $8 / 18(44.4)$ & 0.076 \\
\hline Classification of URTI/cold & $125 / 268(46.6)$ & $71 / 165(43.0)$ & $18 / 33(54.6)$ & 0.225 & $65 / 149(43.6)$ & $10 / 18(55.6)$ & 0.34 \\
\hline Slept under ITN previous night & $210 / 259(81.1)$ & $135 / 178(75.8)$ & $21 / 34(61.8)$ & 0.088 & $130 / 163(79.8)$ & $11 / 19(57.9)$ & 0.38 \\
\hline \multicolumn{8}{|l|}{ Parent/guardian educational level } \\
\hline None & $21(7.37)$ & $16(9)$ & $1(2.7)$ & Reference & $12(7.4)$ & $1(5.3)$ & Reference \\
\hline Primary & $211(74.0)$ & $135(75.8)$ & $24(68.6)$ & 0.30 & $123(75.5)$ & $15(79.0)$ & 0.72 \\
\hline Secondary and above & $53(18.6)$ & $27(15.2)$ & $10(28.6)$ & 0.075 & $28(17.2)$ & $3(15.8)$ & 0.84 \\
\hline \multicolumn{8}{|l|}{ Household socioeconomic status } \\
\hline Least poor quintile & $168(59.0)$ & $107(60.1)$ & 17 (48.6) & \multirow{2}{*}{0.21} & $90(55.2)$ & $13(68.4)$ & \multirow{2}{*}{0.27} \\
\hline Poorest (bottom 80\%) & $117(41.0)$ & $71(39.9)$ & $18(51.4)$ & & $73(44.8)$ & $6(31.6)$ & \\
\hline
\end{tabular}

$\mathrm{IQR}=$ interquartile range; $\mathrm{SD}=$ standard deviation; $\mathrm{URTI}=$ upper respiratory tract infection; ITN = insecticide-treated net

African Republic) found that the sensitivity for ParacheckPf devices was greater than $95 \%$ at a parasite density greater than 500 parasites $/ \mu \mathrm{L} .{ }^{19}$ Both of these evaluations concluded that the use of mRDTs, with reinforced microscopy practice and laboratory quality assurance, would improve the quality of malaria treatment.

The high test agreement between mRDT and microscopy results also supports the previous finding that showed that 97\% of mRDTs are correctly performed and interpreted by CHWs. ${ }^{15}$ While this may have resulted from ongoing training and mentoring related to the $\mathrm{mRDT}$ and rectal artesunate project, it also illustrates that, when they are appropriately trained, community-level CHWs can deliver services of comparable quality to those delivered by facility-level health workers. ${ }^{20}$

There were some limitations for this study; first, although the findings of this study suggested a significantly high clinical response to only antipyretics for children who were mRDT-negative, it is possible that some parents or guardians might have given their sick children medications sourced elsewhere, which might have had an effect on the outcome of the study. Although all enrolled children reportedly did not receive antimalarials within 14 days prior to enrolment, there was no way of corroborating this information. Second, this study was carried out in only 14 village clinics in rural areas of Mchinji District and during the dry season; as such, we may not be able to generalise the results to the entire district, country, or across all seasons. Third, we observed that there was a loss to follow-up of more than $20 \%$ on days 3 and 7 after enrolment. Although this is expected, as caretakers of children whose symptoms resolve may not be motivated to come back to the clinic for review, it may still lead to biased results. Lastly, this study was conducted within a setup where a pilot study on integrating rapid diagnostic testing for malaria and rectal artesunate in iCCM was being carried out. Therefore, the ongoing community campaigns and health promotions on the use of village clinics could have promoted a social desirability bias towards interventions and improved skills for the CHWs, which may not be a true reflection of all CHWs in Malawi.

\section{Conclusions}

Our study has shown that the majority of febrile underfive children with negative mRDT results recovered with only antipyretics. Supportive observation-based supervision and mentoring on the iCCM package for CHWs at the community level should be promoted to help CHWs intervene appropriately, whether an mRDT result is negative or positive, and perform thorough assessments and implement appropriate management to all febrile children.

\section{Acknowledgements}

We recognise the invaluable contributions of all the staff involved in the conduct of this study at Malaria Alert Centre, National Malaria Control Programme (NMCP), IMCI Unit, and Mchinji District Hospital. Sincere thanks to the children and caregivers who participated in the study.

\section{Funding}

This work was supported by the Barr Foundation through Save the Children Malawi, which also reviewed the study design, data collection tools, and data analysis plan.

\section{Competing interests}

All authors declare that they have no competing interests related to this work.

\section{References}

1. WHO | Parasitological confirmation of malaria diagnosis [Internet]. WHO. [cited 2015 Jun 3]. Available from: http://www.who.int/malaria/ publications/atoz/9789241599412/en/

2. Hawkes M, Katsuva JP, Masumbuko CK. Use and limitations of malaria rapid diagnostic testing by community health workers in war-torn Democratic Republic of Congo. Malar J. 2009;8:308.

3. Counihan H, Harvey SA, Sekeseke-Chinyama M, Hamainza B, Banda R, Malambo T, et al. Community Health Workers Use Malaria Rapid Diagnostic Tests (RDTs) Safely and Accurately: Results of a Longitudinal Study in Zambia. Am J Trop Med Hyg. 2012 Jul 2;87(1):57-63.

MMJ VOL 28 (4): December 2016 
4. Thiam S, Thwing J, Diallo I, Fall FB, Diouf MB, Perry R, et al. Scale-up of home-based management of malaria based on rapid diagnostic tests and artemisinin-based combination therapy in a resource-poor country: results in Senegal. Malar J. 2012;11:334.

5. Hopkins H, Asiimwe C, Bell D. Access to antimalarial therapy: accurate diagnosis is essential to achieving long term goals. BMJ. 2009;339:b2606.

6. Abba K, Deeks JJ, Olliaro P, Naing C-M, Jackson SM, Takwoingi Y, et al. Rapid diagnostic tests for diagnosing uncomplicated P. falciparum malaria in endemic countries. Cochrane Database Syst Rev. 2011;(7):CD008122.

7. Hamer DH, Ndhlovu M, Zurovac D, Fox M, Yeboah-Antwi K, Chanda $\mathrm{P}$, et al. Improved diagnostic testing and malaria treatment practices in Zambia. JAMA. 2007 May 23;297(20):2227-31.

8. Uzochukwu BSC, Onwujekwe E, Ezuma NN, Ezeoke OP, Ajuba MO, Sibeudu FT. Improving rational treatment of malaria: perceptions and influence of RDTs on prescribing behaviour of health workers in southeast Nigeria. PloS One. 2011;6(1):e14627.

9. Elmardi KA, Malik EM, Abdelgadir T, Ali SH, Elsyed AH, Mudather MA, et al. Feasibility and acceptability of home-based management of malaria strategy adapted to Sudan's conditions using artemisinin-based combination therapy and rapid diagnostic test. Malar J. 2009;8:39.

10. Chinkhumba J, Skarbinski J, Chilima B, Campbell C, Ewing V, Joaquin MS, et al. Comparative field performance and adherence to test results of four malaria rapid diagnostic tests among febrile patients more than five years of age in Blantyre, Malawi. Malar J. 2010 Jul 20;9(1):209.

11. Ndiaye Y, Ndiaye JLA, Cisse B, Blanas D, Bassene J, Manga IA, et al. Community case management in malaria: review and perspectives after four years of operational experience in Saraya district, south-east Senegal. Malar J. 2013;12:240.

12. NSO. Population and Housing Census 2008 Report. 2008.
13. Mchinji DHO. District Health Management Information System (HMIS) Report 2013. Mchinji, Malawi; 2013.

14. Mchinji DHO. Suspected and confirmed malaria cases for JanuaryOctober 2014.

15. Phiri TB, Kaunda-Khangamwa BN, Bauleni A, Chimuna T, Melody D, Kalengamaliro H, Sande JH, Nsona HK, Mathanga DP. Feasibility, acceptability and impact of integrating malaria rapid diagnostic tests and pre-referral rectal artesunate into the integrated community case management programme. A pilot study in Mchinji district, Malawi. Malar J. 2016;15(1):177. doi:10.1186/s12936-016-1237-2.

16. Government of Malawi, MOH, NMCP. Guidelines for the treatment of malaria in Malawi, 4th Edition, July 2013. GoM,; 2013.

17. Gilroy KE, Callaghan-Koru JA, Cardemil CV, Nsona H, Amouzou A, Mtimuni A, et al. Quality of sick child care delivered by Health Surveillance Assistants in Malawi. Health Policy Plan. 2013 Sep;28(6):573-85.

18. Ruizendaal E, Dierickx S, Peeters Grietens K, Schallig HDFH, Pagnoni F, Mens PF. Success or failure of critical steps in community case management of malaria with rapid diagnostic tests: a systematic review. Malar J. 2014;13:229.

19. Djallé D, Gody JC, Moyen JM, Tekpa G, Ipero J, Madji N, et al. Performance of ParacheckTM-Pf, SD Bioline malaria Ag-Pf and SD Bioline malaria Ag-Pf/pan for diagnosis of falciparum malaria in the Central African Republic. BMC Infect Dis. 2014 Feb 26;14(1):109.

20. Mukanga D, Babirye R, Peterson S, Pariyo GW, Ojiambo G, Tibenderana $\mathrm{JK}$, et al. Can lay community health workers be trained to use diagnostics to distinguish and treat malaria and pneumonia in children? Lessons from rural Uganda. Trop Med Int Health TM IH. 2011 Oct;16(10):1234-42. 